\title{
COMPATIBLE TIGHT RIESZ ORDERS AND PRIME SUBGROUPS
}

\author{
$b y$ N. R. REILLY $\dagger$ \\ (Received 14 February, 1972)
}

Introduction. A tight Riesz group is a partially ordered group which satisfies a strengthened form of the Riesz interpolation property. The term " tight " was introduced by Miller in [8], and the tight interpolation property has been considered in papers by Fuchs [3], Miller [8, 9], Loy and Miller [7] and Wirth [12]. If the closure $\bar{P}$ of the cone $P$, in the interval topology, of such a partially ordered group $G$ contains no pseudozeros, then $\bar{P}$ is itself the cone of a partial order on G. Loy and Miller found of particular interest the case in which this associated partial order is a lattice order. This situation was then considered in reverse by A. Wirth [12] who investigated under what circumstances a lattice ordered group would permit the existence of a tight Riesz order (called a compatible tight Riesz order) for which the initial lattice order is the order defined by the closure of the cone of the tight Riesz order. Wirth gave two fundamental and useful characterizations of those subsets of the cone of a lattice ordered group that can be the strict cone of a compatible tight Riesz order; one is in terms of archimedean classes and the other is an elementwise characterization. Although Loy, Miller and Wirth restricted their attention to abelian groups, much of what they do carries over verbatim to nonabelian groups. In the main result of this paper (Theorem 2.6) a description of the strict cone of a compatible tight Riesz order on a lattice ordered group $G$ is given in terms of the prime subgroups of $G$. This is particularly useful when one is attempting to identify the compatible tight Riesz orders on some particular lattice ordered group or class of lattice ordered groups, since it narrows down to a convenient family of subsets the possible candidates for strict cones of compatible tight Riesz orders. These can then be tested under Wirth's criteria. This technique is illustrated in $\S 5$, where the compatible tight Riesz orders are determined on a lattice ordered group of the type $V\left(\Gamma, G_{y}\right)$, where $\Gamma$ is of finite width, and in $\S 6$, where two examples are considered.

1. By a partially ordered group $(G, \leqq)$ we mean a group $G$ and a partial ordering, $\leqq$, of $G$ which is compatible with the group operation. We shall usually denote the group operation by + , even for nonabelian groups. A partially ordered group $(G, \leqq)$ is called a tight Riesz group if $\leqq$ is a directed partial ordering and if, for any elements $a, b, c, d$ in $G$ such that $a, b<c, d$ there exists an element $x$ in $G$ with

$$
a, b<x<c, d .
$$

The order relation is then called a tight Riesz order. This interpolation property, which is a refinement of the Riesz interpolation property [11], has been extensively considered for abelian partially ordered groups by Miller and Loy in [7], [8] and [9], by Wirth in [12] and has been

$\dagger$ This work was done while the author was a visiting lecturer at Monash University, Melbourne. The author would like to express his gratitude to G. B. Preston for making the visit possible and also to A. Wirth for making available a preliminary draft of [12]. 
called the tight Riesz property. The property is interesting since it ensures that the open interval topology on $(G, \leqq)$ is not discrete [7]. However, we shall not restrict ourselves to abelian groups in this note. For some of the basic properties of tight Riesz groups the reader is referred to [7], [8], [9] and [12].

If $(G, \leqq)$ is a tight Riesz group, then we shall write

$$
P=\{a: a \in G \text { and } 0 \leqq a\}, \quad P^{*}=P \backslash\{0\},
$$

and call $P^{*}$ the strict cone of the tight Riesz order $\leqq$. An element $w \in G$ is a pseudopositive if $w \notin P$ and $w+P^{*} \subseteq P^{*}$. (Since $P^{*}$ is necessarily a normal subset of $G$, this is equivalent to $P^{*}+w \subseteq P^{*}$.) If $w$ and $-w$ are both pseudopositives, then $w$ is a pseudozero. If $G$ has no pseudozeroes, then the open interval topology on $G$ is also Hausdorff and consequently completely regular [7, Theorem 3]. It is also shown by Loy and Miller that $\bar{P}=\{a \in G: a \in P$ or $a$ is a pseudopositive $\}$ is the closure of $P$. Now let $\preccurlyeq$ be another partial ordering of $G$ such that $(G, \preccurlyeq)$ is a lattice ordered group (henceforth $l$-group). We shall write $G^{+}=\{g \in G: 0 \preccurlyeq g\}$ and call $G^{+}$the cone of $(G, \preccurlyeq)$ or of $\preccurlyeq$. If $\bar{P}=G^{+}$, then $\leqq$is a compatible tight Riesz order for $(G, \preccurlyeq)$. For instance, let $\mathbb{R}$ denote the set of real numbers with their usual ordering and $A(\mathbb{R})$ denote the group of all order preserving permutations of $\mathbb{R}$. Let $T=\{\alpha \in A(\mathbb{R}): r<r \alpha$, for all $r \in \mathbb{R}\}$. Then $T$ is the strict cone of a compatible tight Riesz order on $A(\mathbb{R})$. Note that $A(\mathbb{R})$ is also nonabelian.

Let $(G, \preccurlyeq)$ be an $l$-group. Let $T \subset G$. We shall write $x \ll T$ to mean that $n x \preccurlyeq t$ for all positive integers $n$ and all $t \in T$. We shall say that $T$ is a dual ideal of $(G, \preccurlyeq)$ if

(i) $b \preccurlyeq a, b \in T$ implies that $a \in T$;

(ii) $a, b \in T$ implies that $a \wedge b \in T$.

For any proper subset $T$ of $G^{+}$(that is, any subset $T$ such that $T \neq G^{+}, \emptyset$ ) we shall be interested in the following properties:

$T(1) T$ is a dual ideal of $(G, \preccurlyeq)$;

$T(2) T+T=T$;

$T(3) 0 \preccurlyeq x \ll T$ implies that $x=0$;

$T(4) T$ is normal in $G$.

The proof of the following result given by A. Wirth for abelian l-groups is valid without the assumption of commutativity.

Lemma 1.1. (A. Wirth [12]). Let $(G, \preccurlyeq)$ be an l-group and $T$ be a proper subset of $G^{+}$. If $T$ satisfies conditions $T(1), T(2)$ and $T(4)$, then $T$ is the strict cone of a tight Riesz order on $G$. $T$ is the strict cone of a compatible tight Riesz order on $G$ if and only if $T$ satisfies $T(1), T(2)$, $T(3)$ and $T(4)$.

We shall also require the following observations regarding convex $l$-subgroups of $l$-groups. For basic terminology and results relating to $l$-groups the reader is referred to [1] and [4]. 
For any convex subgroup $M$ of a partially ordered group $(G, \leqq)$, we shall denote by $(R(M), \leqq)$ the set of right cosets of $M$ with the naturally induced order, which we also denote by $\leqq$. If $(G, \leqq)$ is actually an l-group and $M$ is a convex $l$-subgroup, then $(R(M), \leqq)$ is a lattice [1].

Lemma 1.2. [1]. For a convex $l$-subgroup $M$ of an $l$-group $(G, \preccurlyeq)$, the following statements are equivalent:

1. $a, b \in G^{+} \mid M$ implies that $a \wedge b \in G^{+} \backslash M$;

2. $a, b \in G^{+} \backslash M$ implies that $a \wedge b \neq 0$;

3. the lattice $(R(M), \preccurlyeq)$ of right cosets of $M$ is totally ordered;

4. the convex $l$-subgroups of $G$ containing $M$ form a chain in the lattice of convex $l$-subgroups of $G$.

A convex $l$-subgroup $M$ of an $l$-group $G$ satisfying the conditions of Lemma 1.2 is called a prime subgroup. These subgroups play a central role in the theory of $l$-groups and particularly in the representation of $l$-groups as groups of order preserving permutations [5].

2. In this section we relate compatible tight Riesz orders to prime subgroups. We begin by providing a source of examples of tight Riesz groups and compatible tight Riesz orders.

A nonempty family $\left\{M_{i}: i \in I\right\}$ of subgroups will be called a normal family if $\bigcup\left\{M_{i}: i \in I\right\}$ is a normal subset of $G$. More generally, when we refer to a family of subgroups $\left\{M_{i}: i \in I\right\}$ we shall always mean a nonempty family.

An l-group $(G, \preccurlyeq)$ is archimedean if, for any $a, b \in G$ such that $n a \preccurlyeq b(n=1,2, \ldots)$, we have $a \leqslant 0$. An archimedean $l$-group is always abelian [4].

The following lemma is almost immediate from Lemmas 1.1 and 1.2.

LemMa 2.1. Let $(G, \preccurlyeq)$ be a divisible l-subgroup. Let $\left\{M_{i}: i \in I\right\}$ be a normal family of prime subgroups such that $T=G^{+} \backslash \bigcup\left\{M_{i}^{+}: i \in I\right\}$ is nonempty. Then $T$ is the strict cone of a tight Riesz order. If $G$ is archimedean, then $T$ is the cone of a compatible tight Riesz order.

Proof. Condition $T(1)$ is satisfied, by Lemma 1.2, since each $M_{i}$ is a prime subgroup. Condition $T(2)$ is satisfied since $G$ is assumed to be divisible. Condition $T(4)$ is satisfied since $\left\{M_{i}: i \in I\right\}$ is assumed to be a normal family. Thus $T$ is the strict cone of a tight Riesz order. If $G$ is archimedean, then $T(3)$ will also be satisfied and then $T$ is the strict cone of a compatible tight Riesz order.

We now weaken the assumption of divisibility of $G$ in Lemma 2.1 to one of denseness of the sets $R\left(M_{i}\right)$ at the cost of being forced to consider only a finite number of primes. We shall say that a partially ordered set $X$ is dense if $x, y \in X$ and $x<y$ implies that there exists a $z \in X$ with $x<z<y$.

Note that, if $\left\{M_{i}: i=1,2, \ldots, n\right\}$ is a family of incomparable primes and $M_{i} \subset \bigcup\left\{M_{j}\right.$ : $j=1,2, \ldots, n, j \neq i\}$, then $M_{i}=M_{j}$, for some $j \neq i$. Otherwise, for each $j \neq i$, we can select $a_{j} \in M_{i}^{+} \backslash M_{j}$. Then $\underset{j}{\vee} a_{j} \in M_{i} \backslash \bigcup\left\{M_{j}: j=1,2, \ldots, n, j \neq i\right\}$.

THEOREM 2.2. Let $(G, \preccurlyeq)$ be an l-group and $\left\{M_{i}: i=1, \ldots, n\right\}$ be a family of proper (that is, $\neq G$ ) prime subgroups of $G$ such that, for each $i, M_{i}$ is normal in $G$ and $\left(R\left(M_{i}\right), \preccurlyeq\right)$ is 
dense. Let $T=G^{+} \backslash \bigcup\left\{M_{i}^{+}: i=1, \ldots, n\right\}$. Then $T$ is the strict cone of a tight Riesz order. If either $G$ is archimedean or each $M_{i}$ is a minimal prime, then $T$ is the strict cone of a compatible tight Riesz order.

Proof. Without loss of generality we assume that the $M_{i}$ are incomparable. Since we have only a finite number of primes, no one of which is equal to $G, T$ is nonempty. Since each $M_{i}$ is a prime subgroup, $T$ clearly satisfies $T(1)$ and, since each $M_{i}$ is normal in $G, T$ satisfies $T(4)$.

Let $t \in T$. Since $t \in G^{+} \mid M_{1}, M_{1} \prec M_{1}+t$. Since $\left(R\left(M_{1}\right), \preccurlyeq\right)$ is dense, there exists an $x_{1}$ such that

$$
M_{1} \prec M_{1}+x_{1} \prec M_{1}+t
$$

and we may (by replacing $x_{1}$ by $\left(x_{1} \vee 0\right) \wedge t$ ) assume that $0 \prec x_{1} \prec t$.

Now suppose that, for $1<j \leqq n$, we have an $x_{j-1}$ such that

and

$$
M_{i} \prec M_{i}+x_{j-1} \prec M_{i}+t \quad(i=1, \ldots, j-1)
$$

$$
0 \prec x_{j-1} \prec t
$$

If

$$
M_{j} \prec M_{j}+x_{j-1} \prec M_{j}+t,
$$

then let $x_{j}=x_{j-1}$. On the other hand, suppose that

$$
M_{j}=M_{j}+x_{j-1} \prec M_{j}+t .
$$

Then, as with $j=1$, since $\left(R\left(M_{j}\right), \preccurlyeq\right)$ is dense, there exists an $x_{j}^{\prime}$ such that

$$
M_{j} \prec M_{j}+x_{j}^{\prime} \prec M_{j}+t
$$

and $x_{j-1} \prec x_{j}^{\prime} \prec t$. For each $i=1, \ldots, j-1$, let $p_{i} \in M_{i}^{+} \backslash M_{j}$. Then, since $M_{j}$ is a prime subgroup, $p=\hat{i}_{i} p_{i} \in \bigcap\left\{M_{i}^{+}: 1 \leqq i \leqq j-1\right\} \backslash M_{j}^{+}$. Let $x_{j}=\left(p+x_{j-1}\right) \wedge x_{j}^{\prime}$. Then, for $i=1, \ldots, j-1$,

and so

$$
\begin{aligned}
M_{i}+x_{j} & =\left(M_{i}+p+x_{j-1}\right) \wedge\left(M_{i}+x_{j}^{\prime}\right) \\
& =\left(M_{i}+x_{j-1}\right) \wedge\left(M_{i}+x_{j}^{\prime}\right) \\
& =M_{i}+x_{j-1} \wedge x_{j}^{\prime} \\
& =M_{i}+x_{j-1}
\end{aligned}
$$

$$
M_{i} \prec M_{i}+x_{j} \prec M_{i}+t
$$

Also,

$$
\begin{aligned}
M_{j}+x_{j} & =\left(M_{j}+p+x_{j-1}\right) \wedge\left(M_{j}+x_{j}^{\prime}\right) \\
& \left.=\left(M_{j}+p\right) \wedge\left(M_{j}+x_{j}^{\prime}\right) \quad \text { (since } M_{j} \text { is normal }\right) \\
& \neq M_{j}
\end{aligned}
$$


since $M \prec M_{j}+p, M_{j}+x_{j}^{\prime}$. Moreover,

$$
0 \prec x_{j}=\left(p+x_{j-1}\right) \wedge x_{j}^{\prime} \preccurlyeq x_{j}^{\prime} \prec t .
$$

Thus

$$
M_{i} \prec M_{i}+x_{j} \prec M_{i}+t
$$

for $i=1, \ldots, j$ and $0 \prec x_{j} \prec t$.

If $M_{j}+x_{j-1}=M_{j}+t$, then we proceed in an analogous manner. Hence, by induction, we can obtain an $x_{n}$ such that

$$
M_{i} \prec M_{i}+x_{n} \prec M_{i}+t
$$

for $i=1, \ldots, n$ and $0 \prec x_{n} \prec t$. Then $\left.x_{n} \in G^{+} \bigcup \bigcup M_{i}^{+}: i=1, \ldots, n\right\}=T$; likewise, $t-x_{n} \in T$ and $t=\left(t-x_{n}\right)+x_{n} \in T+T$. Thus $T$ satisfies condition $T(2)$ and determines a tight Riesz order.

If we now assume in addition that $G$ is archimedean, then $T$ will satisfy condition $T(3)$ and so will be the strict cone of a compatible tight Riesz order.

So finally suppose that each $M_{i}$ is a minimal prime subgroup. Let $0 \preccurlyeq x \ll T$. Then $x \in M_{i}$, for some $i$, say $x \in M_{1} \cap M_{2} \ldots \cap M_{k}$, while $x \notin M_{k+1} \cup M_{k+2} \ldots \cup M_{n}$. For $i=1, \ldots, k$, let $a_{i}$ be such that $a_{i} \notin M_{i}$ and $a_{i} \wedge x=0$ (Such elements exist since each $M_{i}$ is a minimal prime; [1, p. 44]). Let $a=x \vee a_{1} \vee \ldots \vee a_{k}$. Then $a \notin \bigcup\left\{M_{i}: i=1, \ldots, n\right\}$. Thus $a \in T$. Now

$$
\begin{aligned}
2 x & =2 x \wedge a(\text { since } x \ll T) \\
& =2 x \wedge\left(x \vee a_{1} \vee \ldots \vee a_{k}\right) \\
& =(2 x \wedge x) \vee\left(2 x \wedge a_{1}\right) \vee \ldots \vee\left(2 x \wedge a_{k}\right) \\
& =x \vee 0 \vee \ldots \vee 0 \\
& =x .
\end{aligned}
$$

Hence $x=0$ and $T$ satisfies $T(3)$. Thus $T$ is the strict cone of a compatible Riesz order.

In general, for an $l$-group $(G, \preccurlyeq)$ and a family of prime subgroups $\left\{M_{i}: i \in I\right\}, T=$ $G^{+} \backslash \bigcup\left\{M_{i}: i \in I\right\}$ can be the strict cone of a compatible tight Riesz order without the $M_{i}$ being minimal primes. By Lemma 2.1, if $G$ is divisible and archimedean, then $T=G^{+} \backslash M^{+}$will be the strict cone of a compatible tight Riesz order for any proper prime subgroup $M$, minimal or otherwise. An example is given in $\S 6$ where $G$ is not even dense and $T=G^{+} \backslash M^{+}$is the strict cone of a compatible tight Riesz order for some nonminimal prime $M$.

Lemma 2.3. Let $(G, \preccurlyeq)$ be an l-group and $T$ be the strict cone of a compatible tight Riesz order on $G$. Let $S$ be a maximal subsemigroup of $G^{+} \backslash T$. Then $S$ is the cone of a prime subgroup of $G$.

Proof. Clearly $S$ must contain 0 . If $S$ is not convex, then $S^{\prime}=\left\{g: s_{1} \leqq g \leqq s_{2}\right.$, for some $\left.s_{1}, s_{2} \in S\right\}$ will be a subsemigroup of $G^{+}$and, by $T(1)$, will be contained in $G^{+} \backslash T$. This contradicts the maximality of $S$. Hence $S$ is convex and so is the cone of a convex $l$-subgroup $M$ of $G$. To show that $M$ is a prime subgroup, it suffices to show that, if $a, b \in G^{+} \mid S$, then 
$a \wedge b \in G^{+} \mid S$. Suppose that $a, b \in G^{+} \mid S$ and that $a \wedge b \in S$. For $x=a, b$, let $\langle S, x\rangle$ denote the subsemigroup of $G^{+}$generated by $S$ and $x$. By the maximality of $S, A=\langle S, a\rangle \cap T$ and $B=\langle S, b\rangle \cap T$ are both nonempty. Let $x \in A, y \in B$. Since $S$ contains $0, x$ and $y$ are of the form

$$
x=r_{1}+a+r_{2}+\ldots+a+r_{m}
$$

and

$$
y=s_{1}+b+s_{2}+\ldots+b+s_{n}
$$

for some positive integers $m, n$ and some elements $r_{i}, s_{i}$ of $S$. Since $T$ is a dual ideal, $x \wedge y \in T$. On the other hand

$$
\begin{aligned}
x \wedge y & =\left(r_{1}+a+\ldots r_{m}\right) \wedge\left(s_{1}+b+\ldots+s_{n}\right) \\
& \preccurlyeq r_{1} \wedge s_{1}+r_{1} \wedge b+\ldots r_{m} \wedge b+r_{m} \wedge s_{n} \\
& =z, \text { say, }
\end{aligned}
$$

where $z$ is a sum of terms of the form $r_{i} \wedge s_{i}, r_{i} \wedge b, a \wedge s_{i}, a \wedge b$. However, $a \wedge b \in S$ by assumption and the remaining terms are in $S$ by the convexity of $S$. Hence $z \in S$ and so $x \wedge y \in S$, a contradiction. Thus $a \wedge b \notin S$ and $S$ is the cone of a prime subgroup.

COROLLARY 2.4. Let $(G, \preccurlyeq)$ be an l-group and let $T$ be the strict cone of a compatible tight Riesz order on $G$. If $S=G^{+} \backslash T$ is a subsemigroup of $G^{+}$, then $S$ is the cone of a prime subgroup of $G$.

For the next theorem, we need to know that a compatible tight Riesz order $\leqq$ on an l-group $G$ is an isolated order; that is that, if $n a \geqq 0$ for $a \in G$ and some positive integer $n$, then $a \geqq 0$. We make use of the fact that, if $(G, \preccurlyeq)$ is an $l$-group, then $\preccurlyeq$ is an isolated order [4].

Lemma 2.5. Let $(G, \preccurlyeq)$ be an l-group and $T$ be the strict cone of a compatible tight Riesz order $\leqq$. Then $\leqq$ is an isolated order.

Proof. Let $n a>0$, for some positive integer $n$. Without loss of generality, we may assume that $n=2$. Since $\preccurlyeq$ is an isolated order, $a \geqslant 0$. Since $\leqq$ is a tight Riesz order, there exist elements $b^{\prime}, k^{\prime} \in T$ such that

$$
2 a=h^{\prime}+k^{\prime} \text {. }
$$

Let $h=h^{\prime} \wedge k^{\prime}$. Then $h>0$, by $T(1)$, and $2 h \preccurlyeq 2 a$. Thus

$$
0 \preccurlyeq a+a-h-h=a-(a+h-a)+(a-h) .
$$

Let $k=(a+h-a) \wedge h$. Since $a+h-a$ and $h$ are both elements of $T$, so is $k$, by $T(1)$. Now

$$
\begin{aligned}
0 & \preccurlyeq a-(a+h-a)+a-h \\
& \preccurlyeq a-k+a-k \\
& =2(a-k) .
\end{aligned}
$$

Since $\preccurlyeq$ is an isolated order, $a-k \geqslant 0$. Hence $a \geqslant k>0$. So, by $T(1), a \in T$, that is, $a>0$. 
If $n a=0$ for some positive integer $n$, then $a=0$ since the fact that $(G, \preccurlyeq)$ is an $l$-group implies that $G$ is torsion free [4]. Thus $\leqq$ is an isolated order.

THEOREM 2.6. Let $(G, \preccurlyeq)$ be an l-group and $T$ be the strict cone of a compatible tight Riesz order $\leqq$. Then $G^{+} \mid T=\bigcup\left\{S_{i}: i \in I\right\}$, where, for each $i \in I, S_{l}$ is the cone of a prime subgroup.

Proof. From Lemma 2.5, we know that $\leqq$ is an isolated order. Hence, for any $a \in G^{+} \backslash T$, the subsemigroup generated by $a$ is contained in $G^{+} \backslash T$. Thus $G^{+} \backslash T=\bigcup\left\{S_{i}: i \in I\right\}$, where $\left\{S_{i}: i \in I\right\}$ is the set of maximal subsemigroups of $G^{+} \backslash T$. By Lemma 2.3, each $S_{i}$ is the cone of a prime subgroup.

From Theorem 2.6 and Lemma 2.1 we have the following corollary.

COROLlaRY 2.7. Let $(G, \preccurlyeq)$ be a divisible archimedean l-group and $T$ be a nonempty subset of $G^{+} \backslash\{0\}$. Then $T$ is the strict cone of a compatible tight Riesz order if and only if $T=G^{+} \backslash \bigcup\left\{S_{i}: i \in I\right\}$ for some family $\left\{S_{i}: i \in I\right\}$ of cones of prime subgroups of $G$.

THEOREM 2.8. Let $(G, \preccurlyeq)$ be a divisible abelian l-group. Then the following statements are equivalent:

(1) $T$ is the strict cone of a maximal compatible tight Riesz order;

(2) $S=G^{+} \backslash T$ is the cone of a minimal prime subgroup.

Proof. Suppose that (1) holds. By Theorem 2.6, $G^{+} \mid T=\bigcup\left\{S_{i}: i \in I\right\}$, where $\left\{S_{l}: i \in I\right\}$ is the set of maximal subsemigroups of $G^{+} \backslash T$ and each $S_{i}$ is the cone of a prime subgroup $M_{i}$, say. For any fixed $i \in I$, let $P$ be a minimal prime subgroup contained in $M_{i}$. Let $T_{1}=G^{+} \backslash P^{+}$. Since $G$ is divisible, $(R(P), \preccurlyeq)$ is dense, and, since $G$ is abelian, $P$ is normal in $G$. Hence, by Theorem 2.2, $T_{1}$ is the strict cone of a compatible tight Riesz order. But clearly $T \subseteq T_{1}$. Hence $T_{1}=T$ and $G^{+} \mid T=P^{+}$, the cone of a minimal prime subgroup.

Now let (2) hold. By Theorem 2.2, $T$ is the strict cone of a compatible tight Riesz order and, by Theorem 2.6, this order must be a maximal compatible tight Riesz order.

Let $(G, \preccurlyeq)$ be an $l$-group and $g \in G$. By a value of $g$ is meant a convex $l$-subgroup of $G$ which is maximal with respect to the property of not containing the element $g$. A convex $l$-subgroup $M$ of $G$ which is a value for some element of $G$ is called regular. Regular subgroups are prime subgroups [1]. If $M$ is a value of $g \in G$, then $M$ is covered in the lattice of convex $l$-subgroups of $G$ by the convex $l$-subgroup generated by $M$ and $g$. We denote this covering convex $l$-subgroup by $M^{*}$. By Lemma $1.2, M^{*}$ is also a prime subgroup of $G$.

LEMmA 2.9. Let $(G, \preccurlyeq)$ be an abelian l-group and $g \in G^{+}$. Then $g \ll\{t\}$ if and only if $t \in G^{+} \backslash \bigcup\left\{M^{*}: M\right.$ is a value of $\left.g\right\}$.

Proof. Let $t \in G^{+} \backslash \bigcup\left\{M^{*}: M\right.$ is a value of $\left.g\right\}$. Consider any representation of $(G, \preccurlyeq)$ as an $l$-group of order preserving permutations of a set $X$ (for example, the Holland representation [5]). Let $x \in X$ be any element such that $x g \neq x$. Then $g \notin G_{x}=\{a \in G: x a=x\}$. Hence there exists a value $G_{y}$ of $g$ such that $G_{x} \subseteq G_{\gamma}$. Then $G_{x} \subset G_{y}^{*}$ and, since $G_{y}^{*}$ is a prime subgroup of $G$ containing $G_{x}, G_{\gamma}^{*}$ determines a convex congruence $\rho$, say, on $x G$ defined by: $(y, z) \in \rho$ if and only if $y h, z h \in x G_{y}^{*}$ for some $h \in G$ [5, Lemma 4]. The class containing $x$ is $x G_{y}^{*}$. Now $\left(x G_{y}^{*}\right) g=x\left(G_{y}^{*} g\right)=x G_{y}^{*}$ and so $\left(x G_{y}^{*}\right) g^{n}=x G_{y}^{*}$, for any positive integer $n$. On 
the other hand, since $t \notin G_{y}^{*},\left(x G_{y}^{*}\right) t \neq x G_{y}^{*}$ and so, in fact, $\left(x G_{y}^{*}\right) t \cap x G_{y}^{*}=\emptyset$. Since $t \in G^{+}$, we must have $x G_{y}^{*}<\left(x G_{y}^{*}\right) t$. In particular, $x g^{n}<x t$, for all positive integers $n$. Since $x$ was chosen arbitrarily subject to the restriction that $x g \neq x$, we must have $g^{n} \prec t$, for all positive integers $n$. Hence $g \ll\{t\}$.

Now let $g \ll\{t\}$ and let $M$ be a value of $g$. Suppose that $t \in M^{*}$. Then $t \preccurlyeq m+n g$, for some $m \in M$ and some positive integer $n$. Hence

$$
2 t \preccurlyeq 2 m+2 n g \preccurlyeq 2 m+t .
$$

Thus $t \preccurlyeq 2 m$ and $t \in M$. Hence $g \in M$. This is a contradiction, since $M$ is a value of $g$. Therefore $t \notin M^{*}$, for any value $M$ of $g$.

For divisible abelian l-groups we can now give a complete characterization of the strict cone of a compatible tight Riesz order in terms of the prime subgroups.

Proposition 2.10. Let $(G, \preccurlyeq)$ be a divisible abelian l-group. Let $T$ be a nonempty subset of $G^{+} \backslash\{0\}$. Then $T$ is the strict cone of a compatible tight Riesz order if and only if $T=$ $G^{+} \bigcup\left\{M_{i}^{+}: i \in I\right\}$, where each $M_{i}$ is a prime subgroup and for no element $g$ of $G^{+}$is $\bigcup\left\{M^{*}\right.$ : $M$ is a value of $g\} \subseteq \bigcup\left\{M_{i}^{+}: i \in I\right\}$.

Proof. As before, $G$ divisible, abelian and $T=G^{+} \backslash \bigcup\left\{M_{i}^{+}: i \in T\right\}$ nonempty implies that $T$ satisfies $T(1), T(2)$ and $T(4)$. By Lemma 2.9, $T$ satisfies $T(3)$ and so is the strict cone of a compatible tight Riesz order.

Conversely, if $T$ is the strict cone, then, by Theorem 2.6, $T=G^{+} \backslash \bigcup\left\{M_{i}^{+}: i \in I\right\}$ for some prime subgroups $M_{i}$ of $G$. By Lemma 2.9, since $T$ satisfies $T(3)$, there is no element $g \in G^{+}$ such that $\bigcup\left\{M^{*}: M\right.$ is a value of $\left.g\right\} \subseteq \bigcup\left\{M_{i}: i \in I\right\}$ and the proof is complete.

3. This section is devoted to observations regarding convex $l$-subgroups of $l$-groups with compatible tight Riesz orders. Throughout this section, let $(G, \preccurlyeq)$ be an $l$-group and $T$ be the strict cone of a compatible tight Riesz order $\leqq$ on $G$.

LEMMA 3.1. Let $H$ be a subgroup of $G$. The following statements are equivalent:

(1) $H$ is a convex l-subgroup of $(G, \preccurlyeq)$ such that $H \cap T \neq \emptyset$;

(2) $H$ is a convex directed subgroup of $(G, \leqq)$ and $H \neq\{0\}$.

Proof. Let (1) hold and $t \in H \cap T$. Then $H \neq\{0\}$. Since $\preccurlyeq$ is a refinement of $\leqq$ and $H$ is convex with respect to $\preccurlyeq, H$ must be convex with respect to $\leqq$. Let $a, b \in H$. Then

$$
t+(a \vee b)>a \vee b \geqslant a, b .
$$

By Lemma 1.2, $t+(a \vee b)>a, b$ and so $H$ is directed.

Now let (2) hold. Let $a$ be any nonzero element of $H$. If $a>0$, then $a \in H \cap T$. If $a<0$, then $-a \in H \cap T$. If $a$ and 0 are incomparable, then, since $H$ is directed, there exists an element $b \in H$ such that $b \geqq a, 0$. Since $a \geqq 0$, we must have $b>0$ and so $b \in H \cap T$. Hence $H \cap T \neq \emptyset$.

Now let $a \preccurlyeq b \preccurlyeq c$, where $a, c$ are elements of $H$. If $a=c$, then $b=a \in H$. Hence, since $H$ is directed, we may assume that $a<c$. Then

$$
a-(c-a)<a \preccurlyeq b \preccurlyeq c<c+(c-a)
$$


or

$$
2 a-c<b<2 c-a .
$$

Since $H$ is convex with respect to $\leqq, b \in H$ and so $H$ is convex with respect to $\preccurlyeq$. Thus $H$ is a convex directed subgroup of $(G, \preccurlyeq)$ and so is a convex $l$-subgroup.

We shall denote by $\mathscr{C}(G, \preccurlyeq)$ the lattice of convex $l$-subgroups of $(G, \preccurlyeq)$ and by $\mathscr{C}(G, \leqq)$ the set of convex directed subgroups of $(G, \leqq)$.

Corollary 3.2. $\mathscr{C}(G, \leqq)$ is a sublattice of $\mathscr{C}(G, \preccurlyeq)$.

Proof. By Lemma 3.1, we do have that $\mathscr{C}(G, \leqq) \subseteq \mathscr{C}(G, \preccurlyeq)$. Let $H, K \in \mathscr{C}(G, \leqq)$. Then $H, K \in \mathscr{B}(G, \preccurlyeq), H \cap T \neq \emptyset$ and $K \cap T \neq \emptyset$. Hence $(H \vee K) \cap T \neq \emptyset$, where $H \vee K$ is the smallest convex $l$-subgroup of $(G, \preccurlyeq)$ containing $H$ and $K$. Thus $H \vee K \in \mathscr{C}(G, \leqq)$. Now let $h \in H \cap T$, $k \in K \cap T$. By $T(1), h \wedge k \in T$. Thus $h \wedge k \in(\dot{H} \cap K) \cap T$ and $H \cap K \in \mathscr{C}(G, \leqq)$. So $\mathscr{C}(G, \leqq)$ is a sublattice of $\mathscr{C}(G, \preccurlyeq)$.

In general, $\mathscr{C}(G, \leqq)$ need not be a complete sublattice of $\mathscr{C}(G, \preccurlyeq)$. Let $G$ denote the additive group of functions from the set of nonnegative integers $N$ into the additive group of rationals, with the cardinal order. Let $U$ denote an ultrafilter on $N$ containing all subsets $A$ of $N$ such that $|N \backslash A|$ is finite. For $f \in G$, let $N_{f}=\{n: f(n)=0\}$ and let $M=\left\{f \in G: N_{f} \in U\right\}$. Then $G$ is a divisible archimedean $l$-group and $M$ is a prime subgroup. By Lemma $2.1, T=$ $G^{+} \backslash M^{+}$is the strict cone of a compatible tight Riesz order. For $n=2,3, \ldots$, let $H_{n}=$ $\{f \in G: f(n)=0\}$. Then $H=\bigcap\left\{H_{n}: n=2,3, \ldots\right\}=\{f \in G: f(n)=0, n=2,3, \ldots\}$. Thus $H_{n} \in \mathscr{C}(G, \leqq)$, for each $n$, but $H \notin \mathscr{C}(G, \leqq)$.

THEOREM 3.3. The following statements are equivalent:

(1) $M$ is a prime subgroup of $(G, \preccurlyeq)$ such that $M \cap T \neq \emptyset$;

(2) $M \in \mathscr{B}(G, \leqq)$ and $(R(M)$, $\leqq$ is totally ordered.

Proof. Let (1) hold. Then, by Lemma $3.1, M \in \mathscr{C}(G, \leqq)$. Consider any elements $M+x, M+y$ of $R(M)$. It suffices to show that these are comparable in $(R(M)$, $)$. Let $t \in M \cap T$ and, without loss of generality, let $M+x>M+y$. Then $m^{\prime}+x>y$ for some element $m^{\prime} \in M$ and $t+m^{\prime}+x>m^{\prime}+x>y$. If we let $m=t+m^{\prime}$, then, by Lemma $1.2, m+x>y$ and so $M+x>M+y$. Thus $(R(M)$, $)$ is totally ordered.

Conversely, let (2) be satisfied. By Lemma $3.1, M \in \mathscr{C}(G, \preccurlyeq)$ and $M \cap T \neq \emptyset$. Since $\preccurlyeq$ is a refinement of $\leqq$, if $(R(M), \leqq)$ is totally ordered then so must $(R(M), \preccurlyeq)$ be totally ordered. Hence, by Lemma 1.3, $M$ is a prime subgroup of $(G, \preccurlyeq)$.

Another source of subgroups $M$ of $G$ for which $(R(M), \leqq)$ is totally ordered is described in the following theorem.

THEOREM 3.4. Let $M$ be a prime subgroup of $(G, \preccurlyeq)$, and let $T=G^{+} \bigcup\left\{S_{i}: i \in I\right\}$, where each $S_{i}$ is the cone of a prime subgroup $M_{i}$. If either

(1) $M \cap T \neq \emptyset$

or

(2) $M^{+}=S_{j}$, where $S_{j} \ddagger \bigcup\left\{S_{i}: i \neq j\right\}$,

then $(R(M)$, $\leqq$ is totally ordered. 
Conversely, if I is finite and $(R(M), \leqq)$ is totally ordered, then either (1) or (2) holds.

Proof. The assertion that (1) implies that $(R(M), \leqq)$ is totally ordered is just a restatement of one half of Theorem 3.3. So let (2) hold, and let $M+x, M+y$ be any two elements of $R(M)$. It suffices to show that these elements are comparable in $(R(M), \leqq)$, and without loss of generality we may assume that $M+x>M+y$ and $x>y$. Then $x-y \notin S_{j}$. Let $\left.s \in S_{j}\right\rangle$ $\bigcup\left\{S_{i}: i \neq j\right\}$ and $t=s+x-y$. Then $t \geqslant x-y$ and so $t \notin S_{j}$. Also $t \geqslant s$ and so $t \notin \bigcup\left\{S_{i}: i \neq j\right\}$. Hence $t \notin \bigcup\left\{S_{i}: i \in I\right\}$ and so $t \in T$. Thus $t>0$ and $M+x=M+s+x>M+y$. Hence $(R(M), \leqq)$ is totally ordered.

Now suppose that $I$ is finite and that $(R(M), \leqq)$ is totally ordered. If $M^{+}$properly contains $S_{j}$, for any $j$, then, by the maximality of the $S_{i}$ as subsemigroups of $G^{+} \backslash T$, we must have $M \cap T \neq \emptyset$. If $M^{+}=S_{j}$, for any $j$, then we again have the desired conclusion. So suppose that $S_{j} \ddagger M^{+}$, for any $j$. For each $j$, let $s_{j} \in S_{j} \mid M^{+}$. Since $(R(M)$, $)$ is totally ordered, we must have either $M+s_{j}>M$ or $M+s_{j}<M$. But $M+s_{j}<M$ implies that $M+s_{j}<M$, which contradicts the fact that $s_{j} \geqslant 0$. Hence $M+s_{j}>M$ and, for some $a_{j} \in M^{+}, a_{j}+s_{j}>0$ or $a_{j}+s_{j} \in T$. Then $a_{j}+s_{j} \notin S_{j}$ and so $a_{j} \notin S_{j}$. Thus, for each $j$, there exists an $a_{j} \in M^{+} \mid S_{j}$. Hence, if $a$ is the least upper bound of $\left\{a_{j}: j \in I\right\}$ in $(G, \preccurlyeq)$, then $a \in M^{+} \backslash \bigcup\left\{S_{i}: i \in I\right\}$; that is, $a \in M^{+} \cap T$ and $M \cap T \neq \emptyset$.

4. Throughout this section, let $(G, \preccurlyeq)$ be an $l$-group and $H$ be a convex $l$-subgroup of $(G, \preccurlyeq)$. Let $T_{H}(G)$ denote the set of strict cones of compatible tight Riesz orders on $G$ that have nontrivial restrictions to $H$ and let $T(H)$ denote the set of strict cones of compatible tight Riesz orders on $H$. For $T \in T_{H}(G)$, let $T \theta=T \cap H$ and, for $T^{\prime} \in T(H)$, let $T^{\prime} \phi=\{x \in G: x \geqslant h$ for some $\left.h \in T^{\prime}\right\}$.

Let $P_{H}(G)$ denote the set of prime subgroups of $G$ that do not contain $H$, and $P(H)$ denote the set of proper prime subgroups of $H$ (that is, prime subgroups that are not equal to $H$ ). For any $M \in P_{B}(G)$, let $M \sigma=M \cap H$.

LEMMA 4.1. [1]. The mapping $\sigma: M \rightarrow M \sigma$ is a bijection of $P_{H}(G)$ onto $P(H)$. The inverse mapping $\sigma^{-1}$ can be described as follows. For $P \in P(H)$, let $h \in H^{+} \backslash P$. Then

$$
P \sigma^{-1}=\{x \in G:|x| \wedge h \in P\}
$$

where $|x|=x \vee(-x)$. $N^{+} \sigma^{-1}$.

If $M \in P_{H}(G), N \in P(H)$ then we shall also write $M^{+} \cap H=M^{+} \sigma$ and $N \sigma^{-1} \cap G^{+}=$

THEOREM 4.2. If $G$ is an abelian l-group, then $\theta: T \rightarrow T \theta$ is a bijection of $T_{H}(G)$ onto $T(H)$ and $\phi: T^{\prime} \rightarrow T^{\prime} \phi$ is the inverse mapping.

If $T \in T_{B}(G)$ and $T^{\prime} \in T(H)$ are such that

$$
T=G^{+} \backslash \bigcup\left\{S_{i}: i \in I\right\},
$$

where $\left\{S_{i}: i \in I\right\}$ is a family of cones of prime subgroups of $G$ and

$$
T^{\prime}=H^{+} \backslash \bigcup\left\{Q_{j}: j \in J\right\},
$$


where $\left\{Q_{j}: j \in J\right\}$ is a family of cones of prime subgroups of $H$, then

$$
T \theta=H^{+} \backslash \bigcup\left\{S_{i} \sigma: i \in I\right\}
$$

and

$$
T^{\prime} \phi=G^{+} \backslash \bigcup\left\{Q_{j} \sigma^{-1}: j \in J\right\} .
$$

Proof. Let $T \in T_{H}(G)$. Then clearly $T \theta=T \cap H$ satisfies conditions $T(1), T(2)$ and $T(4)$. If $0 \preccurlyeq x \ll T \theta$, then, since $T$ is a dual ideal and $T \theta$ is an ideal of $T, 0 \preccurlyeq x \ll T$. Thus $x=0$ and $T \theta \in T(H)$.

If $T^{\prime} \in T(H)$, then clearly $T^{\prime} \phi\left(=T\right.$, say) satisfies $T(1)$ and $T(4)$ and, since $T^{\prime} \subseteq T, T$ must satisfy $T(3)$. Now let $t \in T$ and $h \in T^{\prime}$ be such that $t \geqslant h$. Then $h=a+b$, for some $a, b \in T^{\prime} \subseteq T$, and so $t=((t-h)+a)+b$. Now $t-h \geqslant 0$ implies that $(t-h)+a \geqslant a$ and so $(t-h)+a, b \in T$. Thus $t \in T+T$ and $T$ satisfies $T(3)$. Finally, $T \cap H=T^{\prime}$ and so $T \in T_{H}(G)$ and $T^{\prime} \phi \theta=T^{\prime}$.

It is clear that $T \theta \phi \subseteq T$, for any $T \in T_{H}(G)$. Let $t \in T$ and $h \in T \theta$. Then $t \wedge h \in T \theta$, $t \wedge h \preccurlyeq t$ and so $t \in T \theta \phi$. Thus $T \theta \phi=T$ and $\theta$ and $\phi$ are inverse mappings.

Let $T$ have the description (A). Then

$$
\begin{aligned}
T \theta=T \cap H & =H^{+} \bigcup\left\{S_{i}: i \in I\right\} \\
& =H^{+} \bigcup\left\{H \cap S_{i}: i \in I\right\} \\
& =H^{+} \bigcup\left\{S_{i} \sigma: i \in I\right\} .
\end{aligned}
$$

Now let $T^{\prime}$ have the description in (B). Let $t \in T^{\prime} \phi$. Then $t \geqslant h$ for some $h \in T^{\prime}$. If $t \in Q_{j} \sigma^{-1}$, for some $j$, then $h \in Q_{j} \sigma^{-1} \cap H=Q_{j} \sigma^{-1} \sigma=Q_{j}$, which contradicts the fact that $h \in T^{\prime}$. Thus $t \notin Q_{j} \sigma^{-1}$, for any $j$, and so $T^{\prime} \phi \subseteq G^{+} \backslash \bigcup\left\{Q_{j} \sigma^{-1}: j \in J\right\}$. Denote this latter set by $R$, say. To obtain the converse inclusion, let $g \in R$ and $h \in T^{\prime}$. Then $h \in T^{\prime} \subseteq T^{\prime} \phi \subseteq R$ and so $h \wedge g \in R$, since each $Q_{j} \sigma^{-1}$ is the cone of a prime subgroup. Thus $h \wedge g \in H^{+} \cap R=H^{+} \backslash \bigcup\left\{Q_{j}: j \in J\right\}=$ $T^{\prime}$. Hence $g \geqslant h \wedge g \in T^{\prime}$ and $g \in T^{\prime} \phi$. Thus $T^{\prime} \phi=R$, as required.

5. In this section we use the description of compatible tight Riesz orders in terms of prime subgroups to help us to identify all the compatible tight Riesz orders on a certain type of abelian $l$-group.

Let $\Gamma$ be a root system, that is, a partially ordered set such that, for any $\gamma \in \Gamma,\{\delta \in \Gamma: \delta \geqq \gamma\}$ is totally ordered. For each $\gamma \in \Gamma$, let $G_{\gamma}$ be a subgroup of the additive group of real numbers and let $P=\Pi\left\{G_{y}: \gamma \in \Gamma\right\}$, the product of the groups $G_{y}$; that is, the additive group of functions $f: \Gamma \rightarrow \bigcup\left\{G_{\gamma}: \gamma \in \Gamma\right\}$ such that $f(\gamma) \in G_{\gamma}$. We shall assume throughout that $G_{\gamma} \neq\{0\}$, for all $\gamma \in \Gamma$. Let $V\left(\Gamma, G_{\gamma}\right)$ denote the subgroup of $P$ consisting of those $v$ in $P$ for which $S_{v}=\{\delta$ : $v(\delta) \neq 0\}$ satisfies the ascending chain condition. If we order $V\left(\Gamma, G_{y}\right)$ by defining $v>0$ to mean that $v(\delta)>0$ for all maximal elements $\delta$ of $S(v)$, then $V\left(\Gamma, G_{y}\right)$ is an abelian $l$-group. By [2], every abelian $l$-group can be imbedded in an $l$-group of the type $V\left(\Gamma, G_{y}\right)$.

We shall say that $\Gamma$ is of width at most $n$ if, for any subset $\Delta$ of $\Gamma$ of incomparable elements, the cardinality of $\Delta$ is less than or equal to $n$.

Throughout this section we let $V=V\left(\Gamma, G_{y}\right)$, where $\Gamma$ is a root system of width at most $n$, and denote the lattice ordering of $V$ introduced above by $\preccurlyeq$. 
For $v \in V$, let $M_{v}$ denote the set of maximal elements of $S_{v}$. Since $\Gamma$ is of width at most $n$, $M_{v}$ must be a finite set.

For each $\delta \in \Gamma$, let $V_{\delta}=\{v: v(\gamma)=0$, for $\gamma \neq \delta\}$. Clearly $V_{\delta}$ is a subgroup of $V$ isomorphic with $G_{\delta}$.

We shall say that a subset $\Delta$ of $\Gamma$ is unbounded above if there is no $\gamma \in \Gamma \backslash \Delta$ such that $\Delta<\gamma$.

LEMMA 5.1. Let $P$ be a proper prime subgroup of $V$. Then

$$
\Delta(P)=\left\{\delta: V_{\delta} \ddagger P\right\}
$$

is a convex totally ordered subset of $\Gamma$ which is unbounded above. Conversely, if $\Delta$ is a convex totally ordered subset of $\Gamma$ which is unbounded above, then

$$
P(\Delta)=\{v \in V: v(\delta)=0, \text { for all } \delta \in \Delta\}
$$

is a proper prime subgroup of $V$. The mappings $P \rightarrow \Delta(P)$ and $\Delta \rightarrow P(\Delta)$ are inverse mappings.

For prime subgroups $P_{1}, P_{2}$ of $V, P_{1} \subseteq P_{2}$ if and only if $\Delta\left(P_{1}\right) \supseteq \Delta\left(P_{2}\right)$ and, consequently, $P$ is a minimal prime subgroup of $V$ if and only if $\Delta(P)$ is a maximal totally ordered subset of $\Gamma$.

Proof. Let $P$ be a proper prime subgroup. For any $\gamma \in \Gamma$ it is clear that either $V_{y} \subseteq P$ or $V_{\gamma} \cap P=\{0\}$. Suppose that $V_{\delta} \subseteq P$, for all $\delta$, and let $v \in V^{+}$. For each $\delta \in M_{v}$, let $u_{\delta} \in V^{+}$ be such that

$$
u_{\delta}(\gamma)=\left\{\begin{array}{l}
v(\delta) \text { if } \gamma=\delta, \\
0 \text { otherwise. }
\end{array}\right.
$$

Then $u_{\delta} \in P$, for each $\delta \in M_{v}$. Hence $\sum 2 u_{\delta} \in P$. But $v \leqslant \sum 2 u_{\delta}$ and so $v \in P$. Thus $V^{+} \subseteq P$, which is a contradiction. Hence $v_{\delta} \neq P$, for some $\delta$. Let $\Delta(P)=\left\{\delta: V_{\delta} \neq P\right\}$. If there are elements $\gamma, \delta \in \Delta(P)$ which are incomparable, then, for any nonzero elements $u_{\gamma} \in V_{\gamma}^{+}, u_{\delta} \in V_{\delta}^{+}$, we have that $u_{\gamma} \wedge u_{\delta}=0$. But $u_{\gamma}, v_{\delta} \notin P$ and so $u_{\gamma} \wedge u_{\delta} \notin P$. Thus we again have a contradiction. Hence $\Delta(P)$ must be totally ordered. Since $P$ is convex, $\Delta(P)$ must be convex and unbounded above.

Now let $\Delta$ be a convex totally ordered subset of $\Gamma$ which is bounded above. Clearly $P(\Delta)$ is a convex $l$-subgroup of $V$. If $a, b \in V^{+} \backslash P(\Delta)$, then $a(\gamma) \neq 0$ and $b(\delta) \neq 0$ for some $\gamma \in M_{a} \cap \Delta$, $\delta \in M_{b} \cap \Delta$. Now $\Delta$ is totally ordered and so, without loss of generality, let $\gamma \leqq \delta$. Then $(a \wedge b)(\gamma)=a(\gamma) \neq 0$. Thus $a \wedge b \notin P(\Delta)$ and so $P(\Delta)$ is a prime subgroup.

Let $P$ be a prime subgroup and $P^{\prime}$ be the prime subgroup of $V$ determined by $\Delta(P)$; $P^{\prime}=\{v \in V: v(\delta)=0$, for all $\delta \in \Delta(P)\}$. Suppose that $v \in V^{+} \backslash P$. Then, for some $\delta \in M_{v}$, $V_{\delta} \neq P$. Thus $M_{v} \cap \Delta(P) \neq \emptyset$. Hence, if $v \in\left(P^{\prime}\right)^{+}$, then $v \in P^{+}$. Conversely, let $v \in P^{+}$and $\delta \in M_{v}$. Define $u_{\delta} \in V$ as follows:

$$
u_{\delta}(\gamma)=\left\{\begin{array}{l}
v(\delta) \text { if } \gamma=\delta, \\
0 \text { otherwise. }
\end{array}\right.
$$

Then $0 \preccurlyeq u_{\delta} \preccurlyeq v$ and so $u_{\delta} \in P \cap V_{\delta}$. Since $V_{\delta}$ is isomorphic with a subgroup of the additive group of real numbers, we must have $V_{\delta} \subseteq P$. Hence $M_{v} \cap \Delta(P)=\emptyset$ and $v(\delta)=0$, for all $\delta \in \Delta(P)$. Thus $v \in\left(P^{\prime}\right)^{+}$and $P=P^{\prime}$. 
If $\Delta$ is a convex totally ordered subset of $\Gamma$ which is unbounded above and $\Delta^{\prime}=\Delta(P(\Delta))$, then, in a similar manner one can show that $\Delta=\Delta^{\prime}$. Thus the mappings $P \rightarrow \Delta(P)$ and $\Delta \rightarrow P(\Delta)$ are inverse mappings, The final assertion of the lemma then follows easily.

To identify the compatible tight Riesz orders on $V$, consider any expression of the form $T=V^{+} \mid \bigcup\left\{P_{i}^{+}: i \in I\right\}$, where $\left\{P_{i}: i \in I\right\}$ is a family of pairwise incomparable prime subgroups. If $|I|=1$, then we also require that $P_{1}$ be a proper prime subgroup. Since $\Gamma$ is of width at most $n$, it follows from Lemma 5.1 that $I$ is finite and that $|I| \leqq n$. Then necessarily $T \neq \emptyset$. Let $I=\{1,2, \ldots, k\}$, where $1 \leqq k \leqq n$, and let $\Delta_{i}=\Delta\left(P_{i}\right)$, in the notation of Lemma 5.1. Since the $P_{i}$ are pairwise incomparable, we must have, for each $j$, that $\Delta_{j} \backslash \cup\left\{\Delta_{i}: i \neq j\right\} \neq \emptyset$. We shall write $\Delta_{j}^{\prime}=\Delta_{j} \bigcup\left\{\Delta_{i}: i \neq j\right\}$. Then $\Delta_{j}^{\prime} \neq \emptyset$, for all $j$. That $T$ satisfies $T(1)$ follows immediately from its description, and $T$ satisfies $T(4)$, since $V$ is abelian.

LEMMA 5.2. The following statements are equivalent:

(1) $T$ satisfies $T(2)$;

(2) $\delta$ minimal in $\Delta$, for some $\Delta \in\left\{\Delta_{i}: i \in I\right\}$, implies that $G_{\delta}$ is dense.

Proof. Let (1) hold and let $\delta$ be minimal in $\Delta$. Then $\delta \in \Delta^{\prime}$. For each $\Delta_{j} \neq \Delta$ let $\delta_{j} \in \Delta_{j}^{\prime}$. Now suppose that $G_{\delta}$ is not dense. Then we may take $G_{\delta}$ to be the additive group of integers. For $j$ such that $\Delta_{j} \neq \Delta$, let $a_{j}$ be any strictly positive element of $G_{\delta_{j}}^{+}$. Let $v \in V$ be the element defined as follows:

$$
v(\gamma)= \begin{cases}1 & \text { if }, \gamma=\delta \\ a_{j} & \text { if } \gamma=\delta_{j} \\ 0 & \text { otherwise. }\end{cases}
$$

Since $\delta, \delta_{j} \in M_{v}$, we have that $M_{v} \cap \Delta_{i} \neq \emptyset$, for all $i$, and hence $v \in T$. However, by considering the $\delta$ th component of $v$ it is clear that there do not exist $x, y \in T$ with $x+y=v$. Thus $T(2)$ is violated, contradicting our initial assumption. Hence $G_{\delta}$ is dense and (1) implies (2).

Now suppose that (2) holds. Let $t \in T, M_{1}=\left\{\delta: \delta \in M_{i} \cap \Delta_{i}\right.$ for some $i \in I$, and $\delta$ is not minimal in $\left.\Delta_{i}\right\}$ and let $M_{2}=\left\{\delta: \delta \in M_{t} \cap \Delta_{i}\right.$ for some $i \in I$, and $\delta$ is minimal in $\left.\Delta_{i}\right\}$. For each $i$ such that $\Delta_{i} \cap M_{1} \neq \emptyset$, let $\delta_{i} \in \Delta_{i}^{\prime}$ be such that $\delta_{i}<\delta$, where $\delta$ is the unique element of $M_{i} \cap \Delta_{i}$, and let $E$ denote the set of such $\delta_{i}$ 's. For $\delta \in E$, let $a(\delta)$ be any strictly positive element of $G_{\delta}$. For $\delta \in M_{2}$, let $a(\delta)$ be any element of $G_{\delta}$ such that $0<a(\delta)<t(\delta)$. Such an element exists, since $\delta$ minimal in $\Delta_{i}$ implies by (2) that $G_{\delta}$ is dense. Now let $a \in V$ be such that

$$
a(\delta)=\left\{\begin{array}{l}
a(\delta), \text { as defined above, if } \delta \in E \cup M_{2}, \\
0 \text { otherwise. }
\end{array}\right.
$$

Then $a \in T, t-a \in T$ and $t=(t-a)+a$. Thus $T(2)$ is satisfied.

LEMMA 5.3. The following statements are equivalent:

(1) $T$ satisfies $T(3)$;

(2) for each i, $\Delta_{i}$ is a maximal (convex) totally ordered subset of $\Gamma$. 
Proof. Suppose that (2) does not hold and that $\Delta_{i}$ is not maximal for some $i$. By Lemma 5.1, $\Delta_{i}$ is unbounded above and is convex. Hence there exists a $\delta$ such that $\delta<\Delta_{i}$. Let $a$ be a strictly positive element of $G_{\delta}$, and define $v \in V$ as follows:

$$
v(\gamma)= \begin{cases}a & \text { if } \gamma=\delta \\ 0 & \text { otherwise }\end{cases}
$$

Let $t \in T$. Then $\Delta_{i} \cap M_{t} \neq \emptyset$ and, if $\delta^{\prime}$ is the unique element of $\Delta_{i} \cap M_{t}$, then $t\left(\delta^{\prime}\right)>0$. Then, since $\delta<\delta^{\prime}$, we have $v \ll t$. Thus (1) does not hold. Hence (1) implies (2).

Suppose that (2) holds and that $0 \prec v \in V$. Let $\delta \in M_{v}$. For each $i \in I$, let $\delta_{j}$ be chosen in $\Delta_{j}^{\prime}$ so that $\delta_{j} \leqq \delta$ if $\delta \in \Delta_{j}$. For each such $\delta_{j}$, let $a\left(\delta_{j}\right)$ be any strictly positive element of $G_{\delta_{j}}$ unless $\delta_{j}=\delta$, in which case let $a\left(\delta_{j}\right)=v(\delta)$. Since each $\Delta_{i}$ is maximal, either $\delta$ is in $\Delta_{j}$ for some $j$, so that $\delta_{j} \leqq \delta$, or $\delta$ is not comparable with any $\delta_{j}$. Thus, if we define $a \in V$ by

$$
a(\gamma)=\left\{\begin{array}{l}
a(\delta) \text { as defined above, if } \gamma=\delta_{j} \text { for some } j, \\
0 \text { otherwise, }
\end{array}\right.
$$

then $2 v \leqslant a$. Thus $T(3)$ holds and (2) implies (1).

THEOREM 5.4. Let $V=V\left(\Gamma, G_{y}\right)$, where $\Gamma$ is of width at most $n(n<\infty)$ and each $G_{y}$ is a nontrivial subgroup of the additive group of real numbers. Let $T \subseteq V^{+}$. Then $T$ is the strict cone of a compatible tight Riesz order if and only if, for some nonempty set $\left\{\Delta_{i}: i \in I\right\}$ of maximal totally ordered subsets of $\Gamma$ such that, if $\delta$ is a minimal element of some $\Delta_{i}$, then $G_{\delta}$ is dense, we have $T=\left\{v \in V:\right.$ for each $i, v(\delta)>0$ for some $\left.\delta \in \Delta_{i}\right\}$.

Proof. Let $T$ be such a strict cone. By Theorem 2.6, $T=V^{+} \backslash \bigcup\left\{P_{i}^{+}: i \in I\right\}$, for some family of prime subgroups $\left\{P_{i}: i \in I\right\}$, and $T \neq \emptyset$. Since $\Gamma$ is of width at most $n$, we may assume that $I$ is finite and that the $P_{i}$ are proper and incomparable. Let $\Delta_{i}=\Delta\left(P_{i}\right)$, as defined in Lemma 5.1. By Lemmas 5.1, 5.2, 5.3, each $\Delta_{i}$ is a maximal totally ordered subset of $\Gamma$ such that $G_{\delta}$ is dense if $\delta$ is minimal in $\Delta_{i}$. From Lemma 5.1 again, we have that $T=V^{+} \bigcup \bigcup\left\{P_{i}^{+}\right.$: $i \in I\}=\left\{v \in V\right.$ : for each $i, v(\delta)>0$ for some $\left.\delta \in \Delta_{i}\right\}$.

Conversely, if $T$ has the description given in the statement of the theorem, then $T=$ $V^{+} \backslash \bigcup\left\{P_{i}^{+}: i \in I\right\}$, where $P_{i}=P\left(\Delta_{i}\right)$ is defined as in Lemma 5.1. By Lemmas 5.2 and 5.3, we then have that $T$ is the strict cone of a compatible tight Riesz order.

For $\Delta$ a maximal totally ordered subset of $\Gamma, P(\Delta)$ is a minimal prime subgroup of $V$, by Lemma 5.1. Thus, for $l$-groups of the type considered in this section and in contrast with the general situation, in order that $T=V^{+} \backslash \bigcup\left\{P_{i}^{+}: i \in I\right\}$, where $\left\{P_{i}^{+}: i \in I\right\}$ is a family of prime subgroups, should be the strict cone of a compatible tight Riesz order on $V$ it is necessary for the $P_{i}$ to be minimal prime subgroups.

6. We conclude with two examples.

Example 1. First we show that it is possible for an l-group $G$ to have compatible tight Riesz orders and yet for there to be no minimal prime subgroup $M$ such that $T=G^{+} \backslash M^{+}$is the strict cone of a compatible tight Riesz order. 
Let $G$ denote the group of functions from the set of nonnegative integers $N$ into the additive group $Z$ of integers with the usual cardinal order, which we shall denote by $\preccurlyeq$. Then $(G, \preccurlyeq)$ is an $l$-group.

Let $U$ be any non-principal ultrafilter on $N$. For each $n \in N, f \in G$, let $A_{n}(f)=\{i \in N$ : $-n \leqq f(i) \leqq n\}$. Let $P=\left\{f \in G\right.$ : for some $\left.n \in N, A_{n}(f) \in U\right\}$. Then $P$ is a non-minimal prime subgroup of $G$. ( $\left(P^{\prime}=\left\{f \in G: A_{0}(f) \in U\right\}\right.$ is also a prime subgroup and $P^{\prime}$ is a proper subgroup of $P$.) Let $T=G^{+} \mid P$. We have immediately that $T$ satisfies condition $T(1)$ since $P$ is a prime subgroup, $T(3)$ since $G$ is archimedean and $T(4)$ since $G$ is abelian. It remains to show that $T(2)$ is satisfied. Let $f \in T$. Define $g, h$ as follows:

$$
\begin{aligned}
g(i) & =\left\{\begin{array}{l}
{[f(i) / 2]+1, \text { for all } i \in N \text { such that } f(i) \neq 0,} \\
0 \text { otherwise, }
\end{array}\right. \\
h & =f-g,
\end{aligned}
$$

where $[x]$ denotes the integral part of the number $x$. Let $A_{n}=\{i: f(i) \geqq n\}, B_{n}=\{i: g(i) \geqq n\}$ and $C_{n}=\{i: h(i) \geqq n\}$. Then, since $f \in T=G^{+} \backslash P$, we have that $A_{n} \in U$ for all $n \in N$, and, in order to show that $g, h \in T$, it is necessary to show that $B_{n}, C_{n} \in U$, for all $n$. Consider $n \geqq 2$. For $i \in A_{2 n-2}$, we have that

$$
f(i) \geqq 2 n-2
$$

and so

$$
f(i) / 2 \geqq n-1 \text {. }
$$

Hence $[f(i) / 2] \geqq n-1$ and

$$
g(i)=[f(i) / 2]+1 \geqq n .
$$

Thus $A_{2 n-2} \subseteq B_{n}$. Since $A_{2 n-2} \in U$ and $U$ is an ultrafilter, $B_{n} \in U$. Since $B_{2} \subseteq B_{0}$ and $B_{2} \subseteq B_{1}$, we have that $B_{n} \in U$, for all $n \in N$. Thus $g \in T$. In a similar way one shows that $h \in T$. Hence $f=h+g \in T+T$ and $T$ satisfies condition $T(2)$. Thus $T$ is the strict cone of a compatible tight Riesz order.

Now let $u$ denote the function such that $u(i)=1$, for all $i$. Then it is easy to see that, for any minimal prime subgroup $M, M+u$ covers $M$ in $(R(M), \preccurlyeq)$. Consequently, for any minimal prime subgroup $M, T=G^{+} \backslash M^{+}$cannot satisfy condition $T(2)$.

Note however that, since $P$ covers $P^{\prime}$ in $\mathscr{C}(G, \preccurlyeq)$ and $P^{\prime}$ is a minimal prime subgroup, $P$ is minimal with respect to the property that $G^{+} \backslash P^{+}$is the strict cone of a compatible tight Riesz order on $G$.

Example 2. This example demonstrates that a dense l-group need not possess a compatible tight Riesz order. This example was considered by Redfield in a different context in [10].

Let $H$ be the $l$-subgroup of the $l$-group $(G, \preccurlyeq)$ in Example 1 consisting of those functions $f: N \rightarrow Z$ for which there is a positive integer $n$ (dependent on $f$ ) such that $f(i)=f(i+n)$, for all $i \in N$. Then $H$ is dense. To see this, let $f \in H^{+}, f \neq 0$, and suppose that $f(i)=f(i+n)$, for 
all $i \in N$. Define $g$ as follows: For any $m \in N$ with $m=r n+s$ for some nonnegative integers $r$, $s$ such that $0 \leqq s<n$, let

$$
g(m)= \begin{cases}f(s)=f(m) & \text { if } r \text { is even, } \\ 0 & \text { if } r \text { is odd }\end{cases}
$$

Then clearly $0 \prec g \prec f$.

Suppose that $T$ is the strict cone of a compatible tight Riesz order. Then, by Theorem 2.6, $H^{+} \backslash T=\bigcup\left\{S_{j}: j \in I\right\}$, where, for each $j \in I, S_{j}$ is the cone of a prime subgroup of $H$. Let $u \in H$ be such that $u(i)=1$, for all $i \in N$.

If $u \notin T$, then $u \in S_{j}$, for some $j \in I$. But $u$ is a strong unit in $H$, since all the functions in $H$ are bounded functions, and so $H^{+} \subseteq S_{j}$. But then $T=\emptyset$, a contradiction.

Hence $u \in T$ and so, by $T(2)$, there exist elements $u_{1}, u_{2}, \ldots$ in $H$ such that

$$
u>u_{1}>u_{2}>\ldots>0 \text {. }
$$

Clearly $\left(u-u_{1}\right) \wedge u_{1}=0$. Hence, for any $j \in I$, either $u-u_{1} \in S_{j}$ or $u_{1} \in S_{j}$. But $u>u_{1}$ implies that $u-u_{1}>0$, that is, that $u-u_{1} \in T$. Hence $u-u_{1} \notin S_{j}$ and so $u_{1} \in S_{j}$. But then $u_{1} \ngtr 0$, a contradiction. Hence there is no compatible tight Riesz order on $H$.

\section{REFERENCES}

1. P. Conrad, Introduction à la théorie des groupes réticulés, Université de Paris, 1967.

2. P. Conrad, J. Harvey and C. Holland, The Hahn embedding theorem for abelian lattice ordered groups, Trans. Amer. Math. Soc. 108 (1963), 143-169.

3. L. Fuchs, Approximation of lattice ordered groups, Ann. Univ. Sci. Budapest Eötvös. Sect. Math. (1965), 187-203.

4. L. Fuchs, Partially ordered algebraic systems (Oxford, 1963).

5. C. Holland, The lattice ordered group of automorphisms of an ordered set, Michigan Math.J. 10 (1963), 399-408.

6. C. Holland, Transitive lattice ordered groups, Math. Zeit. 87 (1965), 420-433.

7. R. J. Loy and J. B. Miller, Tight Riesz groups, J. Australian Math. Soc. 13 (1972), 224-240.

8. J. B. Miller, Higher derivations on Banach algebras, Amer. J. Math, 92 (1970), 301-331. to appear.

9. J. B. Miller, Tight Riesz groups and the Stone-Weierstrass theorem, J. Australian Math. Soc.;

10. R. Redfield, Doctoral Dissertation, Simon Fraser University (1972).

11. F. Riesz, Sur quelques notions fondamentales dans la théorie générale des opérations linéaires, Ann. of Math. 41 (1940), 174-206.

12. A. Wirth, Compatible tight Riesz orders, J. Australian Math. Soc. 15 (1973), 105-111. 